\title{
ENCEPHALOPATHY FOLLOWING INFANTILE GASTRO-ENTERITIS
}

\author{
BY \\ L. CROME \\ From the Department of Neuropathology, the Fountain Hospital, London
}

(RECEIVED FOR PUBLICATION MARCH 3, 1952)

The following case illustrates an unusual neurological hazard of infantile gastro-enteritis.

\section{Case Report}

The patient was the third child in a family free from nervous disease. The first sibling was stillborn. The second and fourth are alive and well.

The patient's early development was normal, and special care was taken to confirm this. It was ascertained that he could hold up his head, smile, reach for objects, grasp them and knock them against the cot. At about the age of 6 months he was beginning to sit up. At that time he was vaccinated and the vaccination is reported to have taken with a moderate local and general reaction. A week later he developed gastro-enteritis and was admitted to hospital in a state of extreme dehydration. Some improvement followed early treatment, but he relapsed again, and remained gravely ill during the following month. Three weeks after admission he developed strabismus and a high-pitched cry. Some twitching of the limbs was noticed and he lapsed into unconsciousness. After that, the gastro-enteritis gradually improved. The cerebrospinal fluid was examined and was found to be normal. On his discharge, four months after admission, he was quadriplegic, blind and characterized as a decerebrate animal. The condition was at that time believed to have been caused by bilateral cortical venous thrombosis.

Soon after his discharge he developed petit mal attacks which were partly controlled by tridione. A report on an electro-encephalogram, taken when he was $1 \frac{1}{2}$ years old, read as follows: 'Severe dysrhythmia in all areas, with no asymmetry. High voltage delta activity is dominant, and occasional spike complexes are seen with no localizing signs.' An air encephalogram showed marked internal hydrocephalus. At about the same time some pallor of the temporal sides was observed in both optic discs and the patient was also noticed to have spasm of the right hand. He did not appear to see although the pupils reacted to light. Involuntary movements of the limbs became more frequent and assumed an athetoid character.

The patient was admitted to the Fountain Hospital at the age of $3 \frac{1}{2}$. After that he was often seen in a position of opisthotonos. The limbs were hypotonic.
The tendon jerks were present and equal. Plantar responses were flexor. He had petit mal attacks and athetoid movements. His mental level was that of a helpless idiot. Two months after admission he had a screaming attack which was followed by marked pallor and death.

\section{Necropsy Report}

The only relevant findings besides those in the central nervous system were in the lungs, which showed on microscopical examination a patchy and varied pattern of lobular collapse, bronchiolitis and catarrhal pneumonia.

The brain weighed $840 \mathrm{~g}$. and presented no obvious naked eye abnormality other than a relatively slight opacity and thickening of the meninges at the base and over the Sylvian fissure. Coronal sections of the brain at the level of the basal ganglia showed a narrowing of the putamen and moderate dilatation of the lateral ventricles.

Histology. Coronal sections of the frontal, parietal, temporal and occipital lobes, and of the basal ganglia, the cerebellum, mid-brain, pons and medulla as well as representative levels of the cord were embedded in celloidin and sections stained with the usual neurological methods. Frozen sections were used for scarlet R staining and for silver impregnation methods.

The meninges and the meningeal blood vessels were somewhat thickened and fibrosed. There was a marked loss of nerve cells in the superficial layers of the cortex and particularly in layer III (Figs. 1 and 2). This loss of nerve cells was both diffuse and focal. It was present in all areas but was most marked in the occipital lobe. Subpial fibrous gliosis was present over most of the cortex, especially in the pit of the sulci. The white matter showed extensive fibrous gliosis, with some loss of myelin in the areas corresponding to the densest gliosis (Fig. 3). The reduction in the size of the putamen was seen to be caused by a narrow area of demyelination and gliosis along a tortuous and dilated vein (Figs. 4 and 5). There was demyelination in the upper part of the pallidum which also showed marked fibrous gliosis and loss of nerve cells. Numerous other areas of fibrous gliosis were present in the central nervous system (Fig. 6). These included the periventricular white matter, the lateral nuclei of the thalami, the red nuclei, the substantia nigra, the reticular formation of the brain stem, 
the white matter of the cerebellum, the peripheral areas and the gray matter of the spinal cord.

A few areas in the cortex of the cerebellum showed atrophy of the granular layer and disappearance of

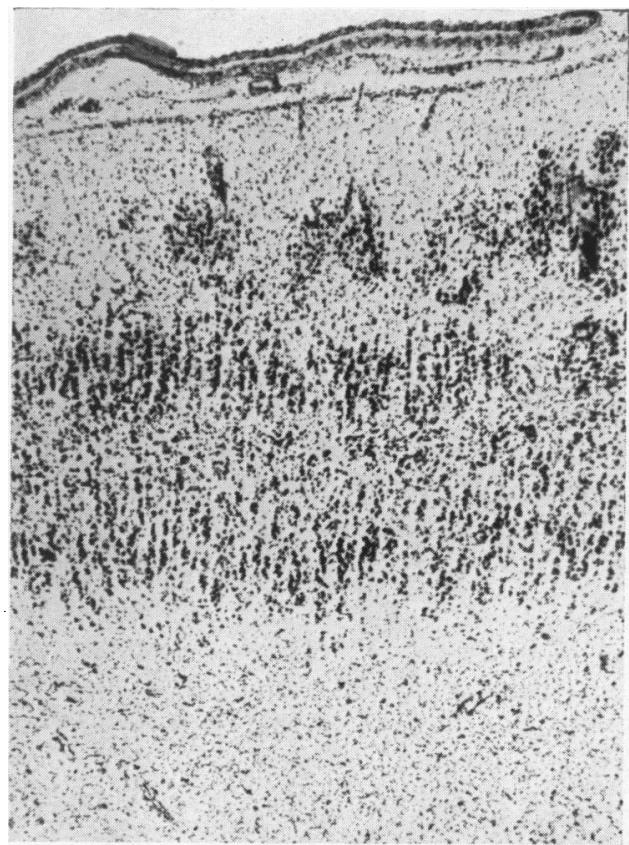

FIG. 1.-Area 18 showing focal and diffuse cell loss in superficial layers. Nissl $\times 35$.

Purkinje cells (Fig. 7). The spinal cord presented, in addition to the gliosis, some marginal pallor of myelin.

No part of the nervous system showed any evidence of persistent active disease other than a small quantity of phagocytosed neutral fat around some of the smaller blood vessels.

\section{Discussion}

The occurrence of marantic thrombosis of the dural sinuses or of the cortical veins is well known. In a chapter of his book dealing with the neurological aspects of dehydration in childhood Ford (1948) enumerates the signs and symptoms of such nervous involvement in the acute stages of the disease. He then adds:

\begin{abstract}
'Actual local lesions of the brain sometimes occur, which give rise to persisting hemiplegia and other cerebral syndromes in children who survive. These are probably the result of thrombosis and infarction.'
\end{abstract}

Infantile gastro-enteritis is not, on the other hand, currently regarded as one of the possible causes of epilepsy or of mental deficiency. It has not been mentioned as such in relation to mental deficiency by Tredgold (1947) or Penrose (1949), or to epilepsy by Bridge (1949). The findings in the present case justify the consideration of the possibility that infantile gastro-enteritis may lead more frequently to lasting neurological sequelae and that the lesions associated with them might be produced in ways other than thrombosis.

It is surprising that a disease so relatively common and severe as infantile gastro-enteritis has not been the object of more enquiry in relation to neurological disease. Further experience might well show that the apparent harmlessness of gastroenteritis in this respect can be explained by the neglect of such study, as well as by the extraordinary capacity of the organically damaged brain to compensate for the defect caused by disease or injury. It may be significant in this connexion that all the cases of familial Schilder's disease described by Meyer and Tennent (1936) had had gastro-intestinal symptoms before the onset of the neurological signs and that a similar association had been observed earlier (Globus, 1932).

It is impossible to be certain of the precise mode of

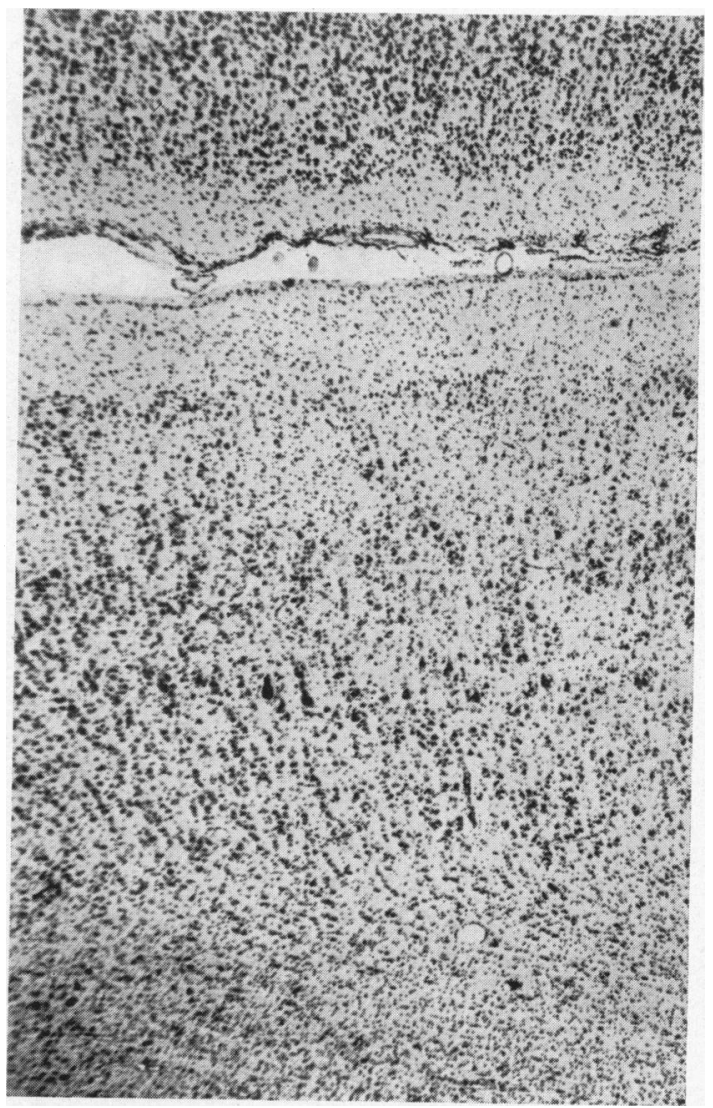

Fig. 2.-Area 4 showing cell loss in superficial layers. $\times 35$. 
Fig. 3.-Occipital lobe showing diffuse fibrous gliosis and partial demyelination. Heidenhain stain, left, and Holzer stain, right.

Fig. 4.-Basal ganglia at level of mamillary bodies. Demyelination of putamen and upper pallidum. Gliosis of striatum. Heidenhain stain, left, and Holzer stain, right.
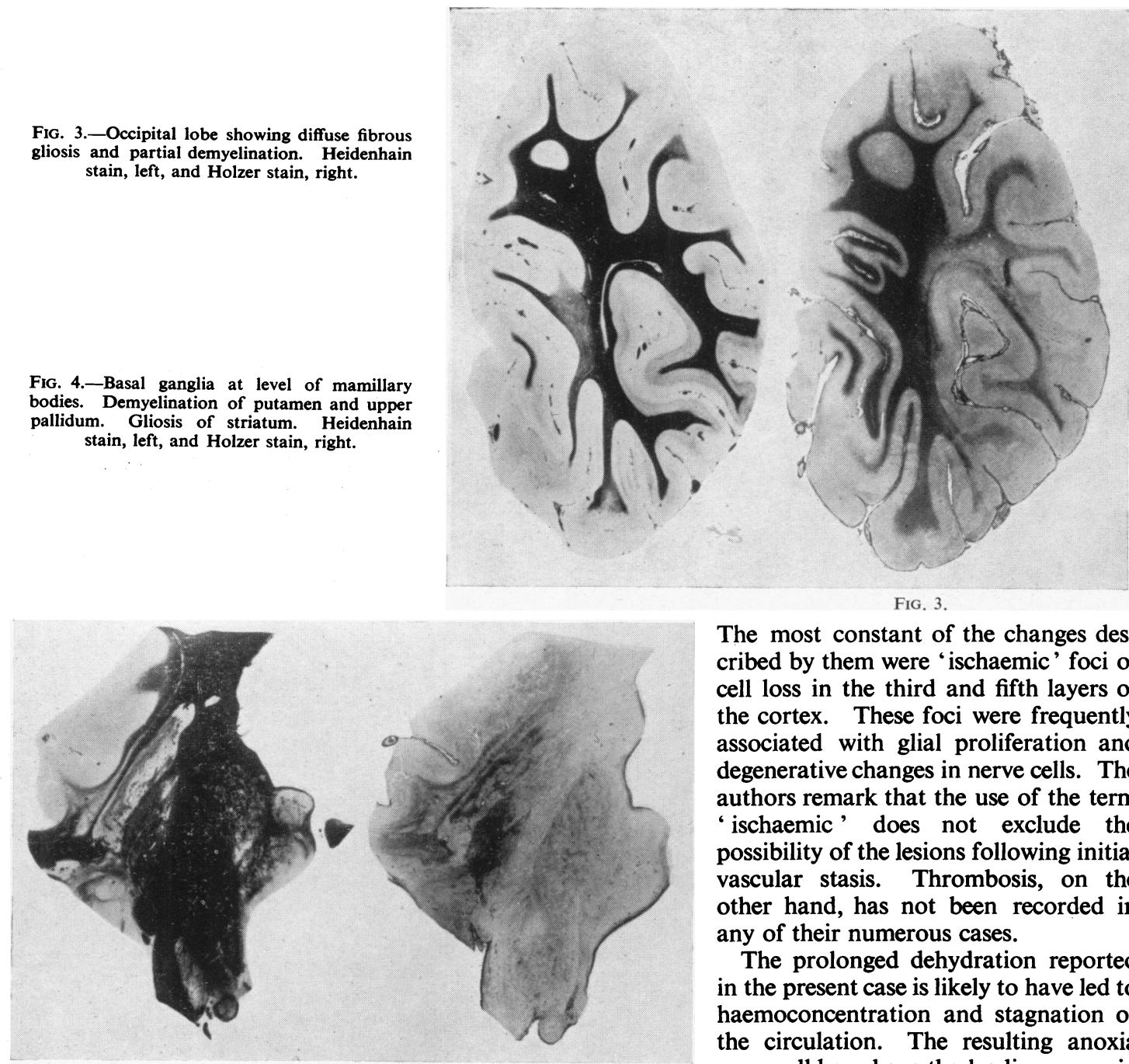

FIG. 4.

production of the structural changes described in this case. They were believed to have been caused by thrombosis. This was not confirmed at necropsy. It is true that the recanalized remnants of a thrombus in one of the sinuses might have been overlooked, but it is much less likely that extensive thromboses of cortical veins had left no histological traces behind them in any of the numerous sections. Meningitis, meningo-encephalitis and post-vaccinial encephalopathy are also unlikely causes in the light of the negative cerebrospinal fluid findings and the nature and distribution of the lesions in the nervous system.

Material from patients dying from bacillary and amoebic dysentery, intestinal tuberculosis and cholera was studied by Alexander and Wu (1935).
The most constant of the changes described by them were 'ischaemic' foci of cell loss in the third and fifth layers of the cortex. These foci were frequently associated with glial proliferation and degenerative changes in nerve cells. The authors remark that the use of the term ' ischaemic' does not exclude the possibility of the lesions following initial vascular stasis. Thrombosis, on the other hand, has not been recorded in any of their numerous cases.

The prolonged dehydration reported in the present case is likely to have led to haemoconcentration and stagnation of the circulation. The resulting anoxia may well have been the leading cause in the production of the lesions. It could have been, of course, only one of many profound functional disturbances operating during the acute stage of the patient's illness. But it has been shown recently by Kerpel-Fronius and his associates (Kerpel-Fronius, Varga, Vönöczky and Kun, 1951) that the disturbances of consciousness in infantile gastro-enteritis and their intensity are related to the degree of circulatory slowing and stagnation anoxia.

It is, perhaps, also necessary to consider the possibility that the epileptic fits by themselves might have produced or contributed to the production of the nervous damage found in the present case. It has been suggested by Scholz (1933 and 1936) that epileptic fits may result in such cerebral and cerebellar lesions as diffuse and patchy gliosis and loss 


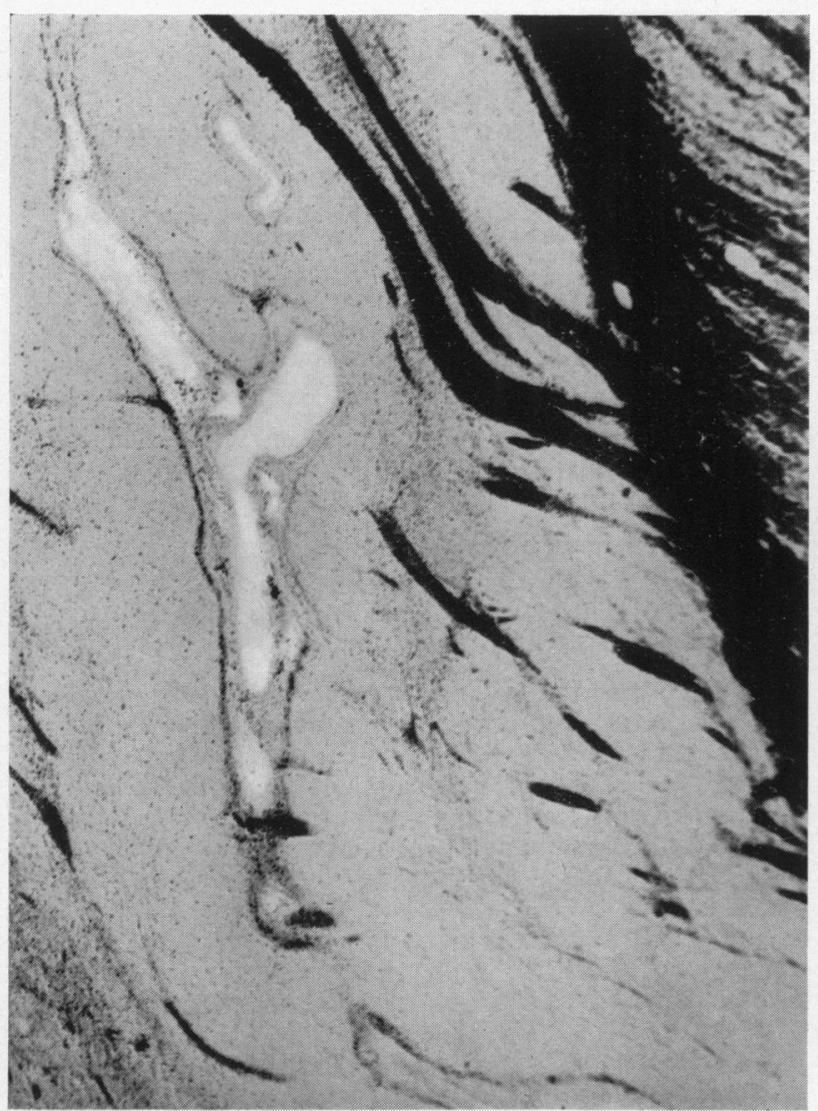

FIG. 5.-Putamen showing demyelination along the course of a tortuous and dilated vein. Heidenhain $\times 35$.

FIG. 6.-Parietal lobe A, and midbrain B, medulla C, and spinal cord D. Holzer stain showing distribution of fibrous gliosis. $\times 2$.

FIG. 7.-Cerebellum with patches of lobular atrophy with rarefaction of granular layer and disappearance of Purkinje cells. Nissl $\times 40$.

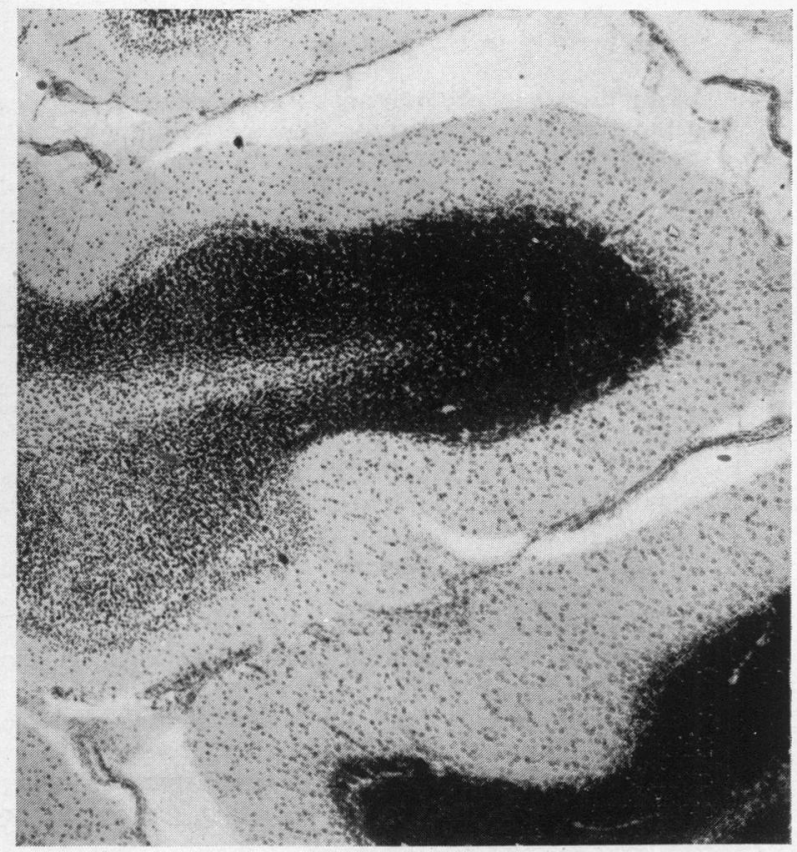

FIG. 7.

FIG. 5.

of nerve cells. While this may be true in certain cases, it is certainly not the general rule and the views of Scholz have met with only guarded and conditional agreement (Meyer, 1939). The experience with the progress of many epileptic children at the Fountain Hospital is also not in accord with this opinion. Mental and neurological deterioration would be expected to set in frequently in cases of major epilepsy in step with the developing lesions. Such deterioration, however, is but rarely seen even in patients suffering from much more severe and frequent fits than the petit mal attacks recorded in the present case.

\section{Summary}

Severe and prolonged infantile gastroenteritis was followed in the present case by quadriplegia, petit mal epilepsy, athetosis, blindness and idiocy. The patient died three years later from respiratory infection. Extensive lesions were found in the central nervous system and are described. They were
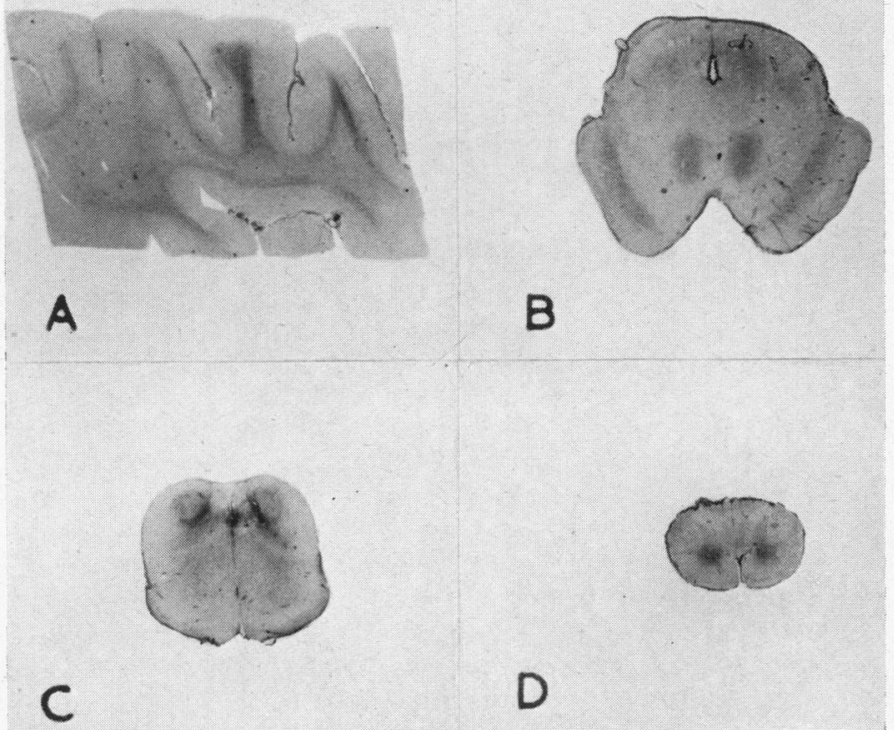

FIG. 6. 
characterized chiefly by a focal and diffuse cell loss in the superficial layers of the cortex. Numerous areas of gliosis and demyelination were also present throughout the nervous system. It is suggested that haemoconcentration with stagnation of the cerebral circulation and the resulting anoxia might have been the main links in the chain of events leading to the production of the lesions.

I am greatly indebted to my lay and medical colleagues at the Fountain Hospital for their cooperation and the use of their case records. Professor A. Meyer and Dr. B. H. Kirman have given much useful advice and criticism. Miss M. F. Craib provided the social and early history of the patient's development. Mr. J. F.
Watt and Mr. J. E. Stevens have assisted in the histological and photographic work.

\section{REFERENCES}

Alexander, L. and Wu, T. T. (1935). Arch. Neurol. Psychiat., Chicago, 33, 72.

Bridge, E. M. (1949). Epilepsy and Convulsive Disorders in Children, New York.

Ford, F. R. (1948). Diseases of the Nervous System in Infancy, Childhood and Adolescence, 2nd ed., p. 758, Springfield.

Globus, J. H. (1932). In Cytology and Cellular Pathology of the Nervous System, ed. W. Penfield: vol. 3, p. 1145, New York.

Kerpel-Fronius, E., Varga, F., Vönöczky, J. and Kun, K. (1951). Helv, paediat. Acta, 6, 377 .

Meyer, A. (1939). J. ment. Sci., 85, 927.

- and Tennent, T. (1936). Brain, 59, 100

Penrose, L. S. (1949). The Biology of Mental Defect. London.

Scholz, W. (1933). Z. ges. Neurol. Psychiat., 145, 471.

(1936). Allg. Z. Psychiat., 104, 89.

Tredgold, A. F. (1947). A Textbook of Mental Deficiency, 7th ed. London.

\section{Scholarships in Aid of Scientific Research}

The Council of the British Medical Association is prepared to receive applications for Research Scholarships, as follows:

An Ernest Hart Memorial Scholarship of the value of $£ 250$

A Walter Dixon Scholarship of the value of $£ 250$

One or More Research Scholarships each of the value of $£ 200$

These scholarships are given to candidates whom the Science Committee of the Association recommends as qualified to undertake research in any subject (including state medicine) relating to the causation, prevention, or treatment of disease.

Each scholarship is tenable for one year, beginning on October 1, 1953. A scholar may be re-appointed for not more than two additional terms. A scholar is not necessarily required to devote the whole of his or her time to the work of research, but may be a member of H.M. Forces or may hold a junior appointment at a university, medical school or hospital, provided the duties of such appointment will not, in the opinion of the Science Committee, interfere with his or her work as a scholar.

Applications for Scholarships must be made not later than March 31, 1953, on the prescribed form, a copy of which will be supplied by the Secretary on application.

Applicants are required to furnish the names of three referees who are competent to speak as to their capacity for the research contemplated. 